\section{Encephalographic Status Epilepticus During Sleep}

Helga Peter ${ }^{1}$ und Thomas Penzel ${ }^{2}$

${ }^{1}$ Marburg, Deutschland

${ }^{2}$ Interdisziplinäres Schlafmedizinisches Zentrum, Charité Universitätsmedizin Berlin, Berlin, Deutschland

\section{Synonyme}

\section{ESES}

\section{Definition}

Eine seltene und ausschließlich während der Stadien des NREM-Schlafs auftretende Störung im EEG; sie ist gekennzeichnet durch kontinuierliche langsame Spike-and-WaveEntladungen, die nach ihrem Auftreten über die Dauer der entsprechenden NREM-Schlafphase anhalten. Dabei sind wenigstens $85 \%$ des NREM-Schlafs durch das Vorkommen dieses epilepsietypischen EEG-Musters gekennzeichnet. Allerdings gehen damit keine klinisch fassbaren Krampfanfälle einher, sodass es sich um eine insgesamt fragwürdige Bezeichnung handelt und ein differentialdiagnostischer Ausschluss epileptischer Erkrankungen erfolgen muss, vor allem bei Kindern.

Siehe auch $\triangleright$,Epilepsie“. 